\title{
AN APPLICATION OF FUZZY GRAPHS TO THE PROBLEM CONCERNING GROUP STRUCTURE
}

\author{
EIJI TAKEDA and TOSHIO NISHIDA, Osaka University \\ (Received September 8, 1975; Revised May 17, 1976)
}

Abstract

A fuzzy graph is utilized to characterize the role played by an individual member in such a group that a class of group members having relationship with any given member has no sharply defined boundary. The concepts of weakening and strengthening points of an ordinary graph presented by Ross and Harary are generalized to those of a fuzzy graph.

\section{Introduction}

The theory of graphs is one of the most important tools in the study of the group structure. For instance, Ross and Harary [4] utilized the graph to characterize such a role of an individual member in a group that: A strengthening member of the group is one whose presence causes the graph corresponding to the group to be more highly connected than that obtained when he is absent, while a weakening member is one whose presence causes the graph to belong to a weaker category of connectedness. Besides this, the graph has been widely utilized to study the problems concerning redundancies, liaison persons, cliques, structural balance and so forth.

In many cases, however, the mere presence or absence of a relation is not adequate to represent a given group structure. As was pointed out in [1], 
there may be different strengths of the relations between individuals. There may even be situations in which it is fuzzy rather than we11-defined whether or not an arbitrary individual has relationship with a given member, that is, a class of group members being in relationship with any given member does not have a sharply defined boundary. In such cases, the ordinary graph may not fully represent the group structure. Instead, the fuzzy graph seems to be a more relevant mathematical model.

The purpose of the present paper is to present an application of the fuzzy graph to the group structure. We shall confine our attention to extending the concepts of weakening and strengthening points of an ordinary graph given by Ross and Harary to those of a fuzzy graph.

In the next section, we shall briefly review the concepts presented by Ross and Harary [4]. In Section 3, we discuss the connectedness of the fuzzy graph. In the final section, the concepts of weakening and strengthening points of a fuzzy graph are introduced and their fundamental properties are investigated.

\section{Weakening and Strengthening Points of a Directed Graph}

To begin with, we will briefly review various kinds of connectedness of directed graphs, or more briefly digraphs [2].

A finite digraph $G$ is strongly connected, or strong, if every two points are mutually reachable; $G$ is unilaterally connected, or unilateral, if for any two points at least one is reachable from the other. We say that $G$ is weakly connected, or weak, if every two points are joined by a semipath. Finally, $G$ is disconnected if it is not even weak. For completeness, we note that a digraph $G$ consisting of exactly one point is strong, for since it does not contain two distinct points, the definition is vacuously satisfied. Let $U_{3}, U_{2}$, $U_{1}$, and $U_{0}$ be collections of all strong digraphs, all unilateral digraphs, all weak digraphs, and all disconnected digraphs, respectively. Obviously we have

$$
U_{3} \subset U_{2} \subset U_{1} \text {. }
$$

In order to divide all digraphs into mutually exclusive connectedness categories, let

$$
C_{3}=U_{3}, \quad C_{2}=U_{2} \backslash U_{3}, \quad C_{1}=U_{1} \backslash U_{2}, \text { and } C_{0}=U_{0}
$$

Then, each digraph belongs to exactly one of the above categories $C_{i}, i=0,1,2,3$. Ross and Harary [4] characterized weakening and strengthening members of a 
group using these disjoint connectedness categories. Let $b$ be any point of a digraph $G$, and let $G_{b}$ be the subgraph obtained from $G$ by the removal of $b$. A point $b$ is said to be of the type $(i, j)$ if $G$ is in $C_{i}$, while $G_{b}$ is in $C_{j}$. The $(i, j)$ point $b$ is called a strengthening point if $i>j$; it is a neutral point if $i=j$; and it is a weakening point if $i<j$. The main results in [4] are the following.

Theorem 1. In any group whatsoever, there are at most two weakening members.

Theorem 2. There are no $(1,3)$ members in any group, and all other $(i, j)$ members can occur.

\section{Connectedness of the Fuzzy Graph}

As was stated in the introduction, one may be concerned with a group where a class of group members being in relationship with any given member is one with an unsharp boundary in which the transition from membership to nonmembership is gradual rather than abrupt. A fuzzy graph may be utilized to represent such a group.

Definition $1[3]$. Let $X$ be a finite set of points $x_{1}, x_{2}, \ldots, x_{n}$, and let $\Gamma$ be the function which associates each point of $X$, say $x_{i}$, with a fuzzy set $\Gamma_{x_{i}}$ in $X$ whose membership function is $\mu_{\Gamma_{x_{i}}}$. Then, $F G=(X, \Gamma)$ is called a fuzzy graph.

In this definition, if each $\mu_{\Gamma_{x_{i}}}, i=1,2, \ldots, n$, takes only two values 0 and 1, $F G$ reduces to an ordinary graph. A more detailed discussion of fuzzy graphs is found in [3].

In order to evaluate the effect of the removal of a point on the connectedness of its fuzzy graph, we introduce

Definition 2. A fuzzy subgraph of $F G=(X, \Gamma)$ is defined to be a fuzzy graph of the form $\left(Y, \Gamma^{\prime}\right)$, where $Y$ is a (non-fuzzy) subset of $X$ and the function $\Gamma^{\prime}$ is defined as

$$
\Gamma_{x_{i}}^{\prime}=\Gamma_{x_{i}} \cap Y \quad \text { for any } x_{i} \varepsilon Y \text {. }
$$


Definition 3. For a fuzzy set $A$ in $X$, with membership function $\mu_{A}$, two fuzzy sets $\Gamma A$ and $\Gamma^{-1} A$ in $X$ are defined by

and

$$
\mu_{\Gamma A}\left(x_{i}\right)=\operatorname{Max}_{x_{j} \in X} \operatorname{Min}\left\{\mu_{A}\left(x_{j}\right), \mu_{\Gamma_{x_{j}}}\left(x_{i}\right)\right\} \text { for a11 } x_{i} \varepsilon X \text {, }
$$
respectively.

$$
\mu_{\Gamma^{-1} A}\left(x_{i}\right)=\underset{x_{j} \varepsilon X}{\operatorname{Max}} \operatorname{Min}\left\{\mu_{A}\left(x_{j}\right), \mu_{\Gamma_{x_{i}}}\left(x_{j}\right)\right\} \text { for a11 } x_{i} \varepsilon X,
$$

We have the following

Proposition 1. Let $A$ and $B$ be two fuzzy sets in $X$, with $\mu_{A}$ and $\mu_{B}$ denoting their respective membership functions. Then,

(a) $\Gamma A \subset \Gamma B$ if $A \subset B$,

(b) $\Gamma^{-1} A \subset \Gamma^{-1} B$ if $A \subset B$,

(c) $\quad \Gamma(A \cap B) \subset \Gamma A \cap \Gamma B$,

(d) $\Gamma^{-1}(A \cap B) \subset \Gamma^{-1} A \cap \Gamma^{-1} B$,

(e) $\quad \Gamma(A \cup B)=\Gamma A \cup \Gamma B$,

(f) $\quad \Gamma^{-1}(A \cup B)=\Gamma^{-1} A \cup \Gamma^{-1} B$.

Proof. Properties (a) and (b) are obvious from Definition 3. Properties

(c) and (d) directly follow from (a) and (b), respectively.

(e)

$$
\begin{aligned}
\mu_{\Gamma(A \cup B)}\left(x_{i}\right) & =\operatorname{Max} \operatorname{Min}\left[\operatorname{Max}\left\{\mu_{A}\left(x_{j}\right), \mu_{B}\left(x_{j}\right)\right\}, \mu_{\Gamma_{x_{j}}}\left(x_{i}\right)\right] \\
& \left.=\underset{x_{j} \varepsilon X}{\operatorname{Max}[\operatorname{Max}} \operatorname{Min}\left\{\mu_{A}\left(x_{j}\right), \mu_{\Gamma_{x_{j}}}\left(x_{i}\right)\right\}, \operatorname{Max}_{j X} \operatorname{Min}\left\{\mu_{B}\left(x_{j}\right), \mu_{\Gamma_{x_{j}}}\left(x_{i}\right)\right\}\right] \\
& =\operatorname{Max}\left[\mu_{\Gamma A}\left(x_{i}\right), \mu_{\Gamma B}\left(x_{i}\right)\right] \\
& =\mu_{\Gamma A \cup \Gamma B}\left(x_{i}\right) .
\end{aligned}
$$

The property (f) is shown in the same way as (e).

Definition 4. For a fuzzy graph $F G=(X, \Gamma)$, the transitive closure of $\Gamma$, denoted by $\hat{\Gamma}$, is defined by

(3.4) $\quad \hat{\Gamma}_{x_{i}}=\left\{x_{i}\right\} \cup \Gamma_{x_{i}} \cup \Gamma_{x_{i}}^{2} \cup \ldots \cup \Gamma_{x_{i}}^{n-1} \quad$ for $x_{i} \varepsilon X$, where $\Gamma_{x_{i}}^{m}=\Gamma\left(\Gamma_{x_{i}}^{m-1}\right), m=2,3, \ldots, n-1$. In the same way, the inverse transitive closure $\hat{\Gamma}^{-1}$ is

(3.5) $\quad \hat{\Gamma}_{x_{i}}^{-1}=\left\{x_{i}\right\} \cup \Gamma_{x_{i}}^{-1} \cup \Gamma_{x_{i}}^{-2} \cup \ldots \vee \Gamma_{x_{i}}^{-n+1}$ for $x_{i} \varepsilon X$,

where $\Gamma_{x_{i}}^{-1}=\Gamma^{-1}\left\{x_{i}\right\}$ and $\Gamma_{x_{i}}^{-m}=\Gamma^{-1}\left(\Gamma_{x_{i}}^{-m+1}\right), m=2,3, \ldots, n-1$. 
We can easily see that

$$
\mu_{\Gamma_{x_{i}}}\left(x_{j}\right)=\mu_{\Gamma_{x_{j}}^{-1}\left(x_{i}\right)} \text { for any } x_{i}, x_{j} \varepsilon X .
$$

The grades of membership $\mu_{\Gamma_{x_{i}}}\left(x_{j}\right)$ and $\mu_{\Gamma_{x_{i}}}^{-1}\left(x_{j}\right)$ may be interpreted as the degree of the existence of a directed path from $x_{i}$ to $x_{j}$ and that from $x_{j}$ to $x_{i}$, respectively. Let us define

(3.7) $\quad \Delta_{x_{i}}=\Gamma_{x_{i}} \cup \Gamma_{x_{i}}^{-1}$ for $x_{i} \varepsilon X$, and

$$
\hat{\Delta}_{x_{i}}=\left\{x_{i}\right\} \cup \Delta_{x_{i}} \cup \Delta_{x_{i}}^{2} \vee \ldots \vee \Delta_{x_{i}}^{n-1} \text { for } x_{i} \varepsilon X \text {, }
$$

where $\Delta_{x_{i}}^{m}=\Delta\left(\Delta_{x_{i}}^{m-1}\right), \quad m=2,3, \ldots, n-1$.

The value of the membership function $\mu_{\Delta_{x_{i}}}\left(x_{j}\right)$ may be interpreted as the degree for two points $x_{i}$ and $x_{j}$ to be joined by a semipath.

With the above preparation, we reach the following

Definition 5. The grades of membership of a fuzzy graph $F G=(X, \Gamma)$ in $U_{3}$, $U_{2}, U_{1}$, and $U_{0}$ are defined by

respectively.

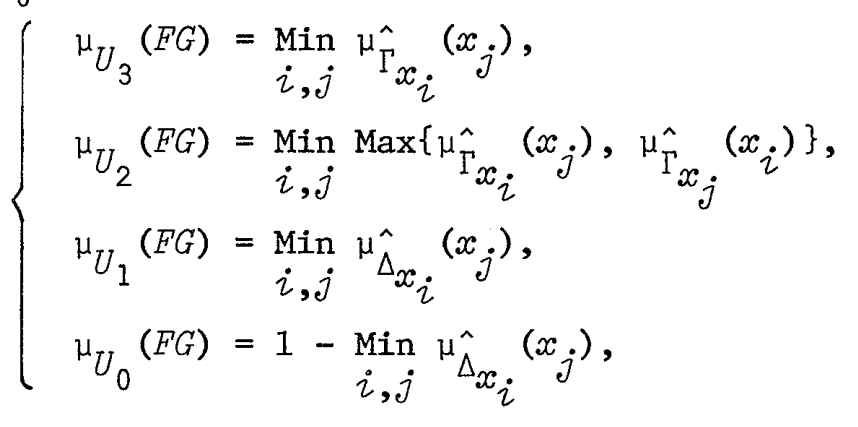

It follows that

$$
\mu_{U_{3}}(F G) \leqq \mu_{U_{2}}(F G) \leqq \mu_{U_{1}}(F G) \quad \text { for any } F G=(X, \Gamma)
$$

Specifically, we can see that for any digraph $G$ in $C_{i}$,

$$
\mu_{U}(G)=0 \text { for } 3 \geqq j>i ; \mu_{U}(G)=1 \text { for } i \geqq j \geqq 1
$$

\section{Weakening and Strengthening Points of a Fuzzy Graph}

In this section we define weakening and strengthening points of a fuzzy graph as a natural extension of those of an ordinary digraph. We then investigate their fundamental properties. 
Definition 6. For a fuzzy graph $F G=(X, \Gamma)$, let $F G_{k}$ be the fuzzy subgraph ( $X \backslash\left\{x_{k}\right\}, \Gamma^{\prime}$ ) obtained from $F G$ by the removal of a point $x_{k}$. Then, the point $x_{k}$ is a weakening point for $U_{i}$ (a $W_{i}$ point, for short) if $\mu_{U_{i}}(F G)<\mu_{U_{i}}\left(F G_{k}\right)$; it is a neutral point for $U_{i}$ (an $N_{i}$ point) if $\mu_{U_{i}}(F G)=\mu_{U_{i}}\left(F G_{k}\right)$; and it is a strengthening point for $U_{i}$ (an $S_{i}$ point) if $\mu_{U_{i}}(F G)>\mu_{U_{i}}\left(F G_{k}\right)$, where $i=1,2,3$.

For instance, a point $x_{k}$, as shown in Figure 1 , is a weakening point for $U_{1}$ because the grade of membership in $U_{1}$ of the fuzzy subgraph $F G_{k}$ is greater than that of $F G$. In the similar way it is also an $S_{2}$ point and an $N_{3}$ point, so we say $x_{k}$ is a point of the type $\left(W_{1}, S_{2}, N_{3}\right)$.

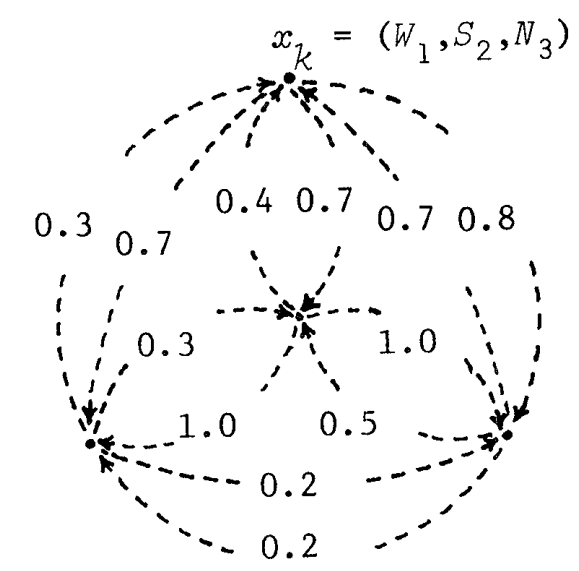

A fuzzy graph $F G$.

$$
\begin{aligned}
& \left(\mu_{U_{1}}(F G), \mu_{U_{2}}(F G), \mu_{U_{3}}(F G)\right) \\
= & (0.8,0.7,0.3) .
\end{aligned}
$$

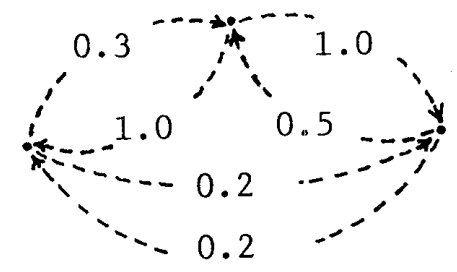

A fuzzy subgraph $F G_{k}$.

$$
\left(\mu_{U_{1}}\left(F G_{k}\right), \mu_{U_{2}}\left(F G_{k}\right), \mu_{U_{3}}\left(F G_{k}\right)\right)
$$$$
=(1.0,0.5,0.3) \text {. }
$$

Figure 1 .

In what follows, for brevity of notation, let

$$
p_{i j}=\mu_{\Gamma_{x_{i}}}\left(x_{j}\right), \quad i, j=1,2, \ldots, n \text {, }
$$

and

$$
q_{i j}=\mu_{\Delta_{x_{i}}}\left(x_{j}\right), \quad i, j=1,2, \ldots, n \text {, }
$$

$$
r_{i j}=\mu_{\Gamma_{x_{i}}}\left(x_{j}\right), \quad i, j \neq k ; i, j=1,2, \ldots, n \text {, }
$$

where $\hat{\Gamma}$ and $\hat{\Delta}$ are respectively as defined in (3.4) and (3.8), and $\hat{\Gamma}^{-}$is the transitive closure of $\Gamma^{\prime}$.

Let $P$ and $Q$ denote respectively $n \times n$ matrices with elements $p_{i j}$ and $q_{i j}$ and let $R$ be an $n \times n$ matrix, whose elements in the $k$-th row and in the $k$-th column are zeros and each $(i, j)$ element is $r_{i j}$, where $i, j \neq k ; i, j=1,2, \ldots, n$. 
The next lemma serves to characterize weakening points for each connectedness category.

Lemma 1. (i) A point $x_{k}$ is a $W_{3}$ point if and only if the elements of $P$ which are equal to $\mu_{U_{3}}(F G)$ are all in the $k$-th row or in the $k$-th column of $P$. (ii) A point $x_{k}$ is a $W_{2}$ point if and only if any $(i, j)$ elements of $P$ such that $\operatorname{Max}\left\{p_{i j}, p_{j i}\right\}=\mu_{U_{2}}(F G)$ are in the $k$-th row and in the $k$-th column of $P$.

(iii) A point $x_{k}$ is a $W_{1}$ point if and only if all the elements of $Q$ which are equal to $\mu_{U_{1}}(F G)$ are in the $k$-th row and in the $k$-th column of $Q$.

Proof. (i) Let $x_{k}$ be a $W_{3}$ point. Suppose that there exists an element, say an $(\tau, m)$ element, $\tau, m \neq k$, which is equal to $u_{U_{3}}(F G)$. Since

we have

$$
r_{i j} \leqq p_{i j}, \quad i, j \neq k ; i, j=1,2, \ldots, n \text {, }
$$

$$
\mu_{U_{3}}\left(F G_{k}\right)=\operatorname{Min}_{i, j \neq k}\left\{r_{i j}\right\} \leqq p_{Z m}=\mu_{U_{3}}(F G)
$$

which contradicts the assumption that $x_{k}$ is a $W_{3}$ point. Therefore, every element of $P$ which is equal to $\mu_{U_{3}}(F G)$ is in the $k$-th row or in the $k$-th column of $P$.

Conversely, assume that the elements of $P$ which are equal to $\mu_{U_{3}}(F G$ ) are all in the $k$-th row or in the $k$-th column of $P$. First, notice that if an element which is equal to $\mu_{U_{3}}(F G)$ is in the $k$-th row ( column) of $P$, then every non-diagonal element in the $k$-th row ( column) of $P$ is equal to $\mu_{U_{3}}(F G)$. Hence

which yie1ds

$$
\operatorname{Min}\left\{p_{i k}, p_{k j}\right\}=\mu_{U_{3}}(F G)<p_{i j}, i, j \neq k ; i, j=1,2, \ldots, n,
$$

$$
r_{i j}=p_{i j}>\mu_{U_{3}}(F G), \quad i, j \neq k ; i, j=1,2, \ldots, n .
$$

Therefore

$$
\mu_{U_{3}}\left(F G_{k}\right)=\operatorname{Min}_{i, j \neq k}\left\{r_{i j}\right\}>\mu_{U_{3}}(F G) \text {, }
$$

so that $x_{k}$ is a $W_{3}$ point, which completes the proof of (i).

The proofs of (ii) and (iii) are similar to that of (i).

The following theorem is an immediate consequence of Lemma 1.

Theorem 3. There exist at most two $W_{i}$ points in any fuzzy graph, where $i=1,2,3$. Further, any fuzzy graph with $n(n \geqq 3)$ points has at most one $W_{1}$ 
$\left(W_{3}\right)$ point.

Lemma 2. For any fuzzy graph $F G=(X, \Gamma)$, there exists a path $\left\{x_{i_{1}}, x_{i_{2}}, \ldots\right.$, $\left.x_{i_{s}}\right\}(s \geqq n)$ such that:

(1) every point of $X$ appears in the path;

(2) $\mu_{\Gamma_{x_{i}}}\left(x_{i_{Z+1}}\right) \geqq \mu_{U_{2}}(F G), \quad \tau=1,2, \ldots, s-1$.

Proof. Let us construct an ordinary digraph $G=\left(X, \Gamma^{\prime \prime}\right)$ from $F G$ as follows:

$$
\mu_{\Gamma_{i}^{\prime \prime}}\left(x_{j}\right)\left\{\begin{array}{c}
1 \\
0
\end{array}\right\} \text { according as } p_{i j}\left\{\begin{array}{l}
\geqq \\
<
\end{array}\right\} \mu_{U_{2}}(F G), \quad i, j=1,2, \ldots, n \text {. }
$$

Since $\operatorname{Max}\left\{p_{i j}, p_{j i}\right\} \geqq \mu_{U_{2}}(F G), G$ includes a tournament as a partial graph of $G$. Since every tournament has a Hamiltonian path, $G$ has a Hamiltonian path. On the other hand, we can easily see from Definition 4 that if $p_{i j} \geqq \mu_{U_{2}}(F G)$, then there exists at least a path $\left\{x_{i}, x_{u}, \ldots, x_{v}, x_{j}\right\}$ such that

$$
\begin{aligned}
\mu_{\Gamma_{x_{i}}}\left(x_{u}\right) & \geqq \mu_{U_{2}}(F G), \\
& \vdots \\
\mu_{\Gamma_{x_{v}}}\left(x_{j}\right) & \geqq \mu_{U_{2}}(F G) .
\end{aligned}
$$

Thus, we obtain the desired result.

The following theorem shows that in any fuzzy graph with $n(n \geqq 2)$ points, it is impossible for all points to be strengthening ones for $U_{2}\left(U_{1}\right)$.

Theorem 4. In any fuzzy graph $F G$ with $n(n \geq 2)$ points, there exist at least two points which are either weakening or neutral ones for $U_{2}\left(U_{1}\right)$.

Proof. Let a path $\left\{x_{i_{1}}, x_{i_{2}}, \ldots, x_{i_{s}}\right\}$ satisfy (1) and (2) of Lemma 2 . Without loss of generality, we can assume that the initial and final points $x_{i_{1}}$ and $x_{i}$ appear exactly once in the path. For, if the initial point (the final point) appears more than once in the path, we can delete the first point ( the last point) of the path, so that the remaining path also meets the requirements (1) and (2).

Now, according to the above assumption, a path $\left\{x_{i_{2}}, x_{i_{3}}, \ldots, x_{i_{s}}\right\}$ and a path $\left\{x_{i_{1}}, x_{i_{2}}, \ldots, x_{i_{s-1}}\right\}$ contain respectively all points in $X \backslash\left\{x_{i_{1}}\right\}$ and all points in $X \backslash\left\{x_{i_{s}}\right\}$. Therefore

and

$$
\mu_{U_{2}}\left(F G_{i_{1}}\right) \geqq \mu_{U_{2}}(F G) \text {, }
$$




$$
\mu_{U_{2}}\left(F G_{i_{s}}\right) \geqq \mu_{U_{2}}(F G)
$$

Thus, each of $x_{i_{1}}$ and $x_{i_{s}}$ is either a $W_{2}$ or $N_{2}$ point, which completes the proof for $U_{2}$.

The proof for $U_{1}$ is similar.

Corollary 1. Any fuzzy graph with $n(n \geq 3)$ points has at least one $N_{1}$ point.

Proof. It is an immediate consequence of Theorems 3 and 4.

Theorem 5. If a fuzzy graph $F G$ with $n(n \geqq 3)$ points has two $W_{2}$ points, then

$$
\mu_{U_{2}}(F G)<\mu_{U_{1}}(F G) .
$$

Proof. Let $x_{k}$ and $x_{l}$ be $W_{2}$ points, i.e.,

(4.4) $\quad \mu_{U_{2}}\left(F G_{k}\right)>\mu_{U_{2}}(F G)$,

and

$$
\text { (4.5) } \quad \mu_{U_{2}}\left(F G G_{Z}\right)>\mu_{U_{2}}(F G) \text {. }
$$

Suppose that

$$
\mu_{U_{2}}(F G)=\mu_{U_{1}}(F G) \text {. }
$$

From (3.10) and (4.4) through (4.6), we find that $x_{k}$ and $x_{l}$ must be $W_{1}$ points, which contradicts Theorem 3. This completes the proof.

Theorem 6. Any $W_{3}$ point is either a $W_{2}$ one or an $N_{2}$ one.

Proof. Let $x_{k}$ be a $W_{3}$ point. From the proof of Lemma 1, we get

$$
r_{i j}=p_{i j}, \quad i, j \neq k ; i, j=1,2, \ldots, n \text {. }
$$

Therefore,

$$
\mu_{U_{2}}\left(F G_{k}\right) \geqq \mu_{U_{2}}(F G)
$$

which ends the proof.

The following theorem directly follows from Definition 6 and (3.10).

Theorem 7. If $\mu_{U_{i}}(F G)=\mu_{U_{j}}(F G)$ for some $i<j$, then an $S_{i}$ point is also an $S_{j}$ point.

Theorem 8. If $\mu_{U_{i}}(F G)=\mu_{U_{j}}(F G)$ for some $i>j$, then a $W_{i}$ point is also a $W_{l}$ point, where $1 \leqq Z \leqq i$. 
Proof. Let $i=3$ and $j=1, i . e .$,

$$
\mu_{U_{3}}(F G)=\mu_{U_{1}}(F G) \text {. }
$$

Let $x_{k}$ be a $W_{3}$ point. From (3.10) and (4.7) we obtain

and

$$
\mu_{U_{2}}\left(F G_{k}\right)>\mu_{U_{2}}(F G) \text {, }
$$

$$
\mu_{U_{1}}\left(F G_{k}\right)>\mu_{U_{1}}(F G) .
$$

Thus, $x_{k}$ is a $W_{l}$ point, where $1 \leqq l \leqq 3$. Next, assume that $x_{k}$ is a $W_{2}$ point and that $\mu_{U_{2}}(F G)=\mu_{U_{1}}(F G)$. It follows that

$$
\mu_{U_{1}}\left(F G_{k}\right)>\mu_{U_{1}}(F G) \text {. }
$$

Thus, $x_{k}$ is a $W_{l}$ point, where $1 \leqq Z \leqq 2$. Finally, we shall prove that if $x_{k}$ is a $W_{3}$ point and $\mu_{U_{3}}(F G)=\mu_{U_{2}}(F G)$ then it is a $W_{2}$ point, where $1 \leqq \tau \leqq 3$. Since it is obvious that $x_{k}$ is a $W_{2}$ point, it suffices to show that $x_{k}$ is a $W_{1}$ point. Using Lemma 1 , it follows that both in the $k$-th row and in the $k$-th column of $P$ there exists an element which is equal to $\mu_{U_{3}}(F G)$. Hence we get from the proof of Lemma 1

Thus we have

$$
p_{k j}=p_{j k}=\mu_{U_{3}}(F G), \quad j \neq k ; j=1,2, \ldots, n .
$$

$$
\mu_{\Gamma_{x_{k}}} \cup \Gamma_{x_{k}}^{-1\left(x_{j}\right) \leq \mu_{U_{3}}}(F G), \quad j \neq k ; j=1,2, \ldots, n,
$$

which yields

Therefore we get

$$
q_{k j}=q_{j k} \leqq \mu_{U_{3}}(F G), \quad j \neq k ; j=1,2, \ldots, n .
$$

so that

$$
\mu_{U_{1}}(F G)=\mu_{U_{3}}(F G)
$$

$$
\mu_{U_{1}}\left(F G_{k}\right)>\mu_{U_{1}}(F G) .
$$

This completes the proof.

Theorem 9. Let $x_{k}$ be a $W_{i}$ point. If $\mu_{U_{i}}\left(F G_{k}\right)=\mu_{U_{j}}\left(F G_{k}\right)$ for some $i<j$, Then $x_{k}$ is also a $W_{l}$ point, where $1 \leqq \tau \leqq j$.

Proof. The proof of this theorem is similar to that of Theorem 8 .

In closing, we shall show how results of Ross and Harary can be obtained from our results as the special cases. First, note that, in the case of the ordinary digraph $G, \mu_{U_{i}}\left(G_{k}\right)>\mu_{U_{i}}(G)$ if and only if $\mu_{U_{i}}\left(G_{k}\right)=1$ and $\mu_{U_{i}}(G)=0$, that is, $G_{k} \in U_{i}$ and $G \notin U_{i}$. With the understanding that a weakening point for $U_{0}$ is one whose presence makes its fuzzy graph more highly disconnected than it 
would be without the point, the $W_{0}$ point is defined to be the $W_{1}$ point. We can easily see from Theorem 3 that any digraph has at most two weakening points. And, from Theorem 8 , we can find that there are no $(1,3)$ points in any digraph.

\section{References}

[1] Harary,F.,"Graph Theoretic Methods in the Management Sciences," Management Science, 5 (1959), 387-403.

[2] Harary,F., R.Z.Norman and D.Cartwright, Stmuctural Models: An Introduction to the Theory of Directed Graphs, John Wiley \& Sons, Inc., New York, 1965.

[3] Kaufmann,A., Introduction to the Theory of Fuzzy Subsets, Vol. 1, Academic Press, New York, 1975.

[4] Ross,I.C., and F.Harary, "A Description of Strengthening and Weakening Members of a Group," Sociometry, 22 (1959), 139-147.

[5] Zadeh,L.A., "Fuzzy Sets," Information and Control, 8 (1965), 338-353.

Eiji TAKEDA and Toshio NISHIDA

Department of Applied Physics

Faculty of Engineering

Osaka University

Yamada-Kami, Suita

Osaka, 565, Japan 